DOI: $10.17516 / 1999-494 X-0274$

УДК 623.775:623.4.011

\title{
Methods for Evaluating the Effectiveness \\ of Aerosol Ormations in Protecting Objects against Aimed Fire from a Grenade Launcher
}

\author{
Yuriy L. Koziratsky* and Dmitry V. Prokhorov \\ Military Educational and Scientific Center of Air Forces \\ «Air Force Academy named after N.E. Zhukovsky \\ and Yu.A. Gagarin» \\ Voronezh, Russian Federation
}

Received 30.08.2020, received in revised form 12.09.2020, accepted 01.10.2020

Abstract. With the use of graph theory, probability theory and Laplace transform methods, the methods have been developed for evaluating the effectiveness of aerosol formations to protect objects against aimed fire from a grenade launcher. The methods enable to obtain probabilistic indicators of shells hitting the target when firing from a grenade launcher in difficult conditions, taking into account the time characteristics of the firing process and a set of aiming errors caused by a number of factors, including the use of aerosol screen.

Keywords: aerosol formation, aimed fire, protection of objects, evaluation of effectiveness.

Citation: Koziratsky Yu.L., Prokhorov D.V. Methods for evaluating the effectiveness of aerosol ormations in protecting objects against aimed fire from a grenade launcher, J. Sib. Fed. Univ. Eng. \& Technol., 2020, 13(7), 882-893. DOI: 10.17516/1999494X-0274

(C) Siberian Federal University. All rights reserved

This work is licensed under a Creative Commons Attribution-Non Commercial 4.0 International License (CC BY-NC 4.0).

* Corresponding author E-mail address: urleo@bk.ru 


\title{
Методика оценки эффективности
}

\section{применения аэрозольных образований \\ для защиты объектов от прицельной стрельбы}

\section{из гранатомета}

\author{
Ю.Л. Козирацкий, Д.В. Прохоров \\ Военный учебно-научный центр Военно-воздушных сил \\ «Военно-воздушная академия \\ имени профессора Н.Е. Жуковского и Ю.А. Гагарина» \\ Российская Федераиия, Воронеж
}

Аннотация. С использованием теории графов, теории вероятностей, методов преобразования Лапласа разработана методика оценки эффективности применения аэрозольных образований для защиты объектов от прицельной стрельбы из гранатомета. Методика позволяет получать вероятностные показатели попадания снарядов в цель при стрельбе из гранатомета в сложных условиях обстановки с учетом временных характеристик процесса стрельбы и совокупности ошибок прицеливания, обусловленных рядом факторов, в том числе применением аэрозольных завес.

Ключевые слова: аэрозольное образование, прицельная стрельба, защита объектов, оценка эффективности.

Цитирование: Козирацкий, Ю.Л. Методика оценки эффективности применения аэрозольных образований для защиты объектов от прицельной стрельбы из гранатомета / Ю.Л. Козирацкий, Д.В. Прохоров // Журн. Сиб. федер. ун-та. Техника и технологии, 2020. 13(7). С. 882-893. DOI: 10.17516/1999-494X-0274

\section{Введение}

Анализ войн и вооруженных конфликтов последних лет показывает, что одним из эффективных способов защиты объектов, вооружения и военной техники (ВВТ) от поражения различными боеприпасами является применение аэрозольных завес. Разработке моделей и методик оценки их эффективности посвящено множество работ, в том числе с участием авторов [1-4]. Однако вопрос оценки точности ведения прицельной стрельбы стрелком из гранатомета по цели в случае прикрытия ее аэрозольным образованием (АО) остается нераскрытым.

Поэтому целью работы стала разработка методики оценки эффективности применения аэрозольных образований для защиты объектов от прицельной стрельбы из гранатомета.

\section{Постановка задачи}

Действительность стрельбы зависит от способа ведения огня, дальности стрельбы, характера цели, условий наблюдения, степени обученности стрелка и ряда других причин [5]. При ведении боевых действий (например, нападение на колонну техники на марше) может возникать ситуация, когда гранатометчик, осуществляя подготовку к выстрелу по цели, внезапно теряет ее из виду вследствие постановки аэрозольной завесы, прикрывающей цель. В этих условиях стрелок вынужден произвести выстрел в цель, находящуюся в некоторой области нео- 
пределенности, обусловленной наиболее вероятным нахождением ее по представлению стрелка. Стрельба при этом будет осуществляться по памяти, в направлении шума мотора или лязга гусениц [6], опираясь на какие-либо визуальные ориентиры. Ввиду высокой плотности завесы и значительных флуктуаций коэффициента пропускания аэрозольного образования [1-4] такими ориентирами для стрелка могут стать контрастные выбросы, обусловленные флуктуациями АО. Вследствие случайного характера этих выбросов точка прицеливания (ТП) стрелка также будет формироваться по случайному закону с ошибками, обусловленными опытностью и памятью стрелка, а также флуктуационными свойствами АО. Геометрия задачи представлена на рис. 1. Будем считать также, что стрельба осуществляется одним стрелком (последовательно)

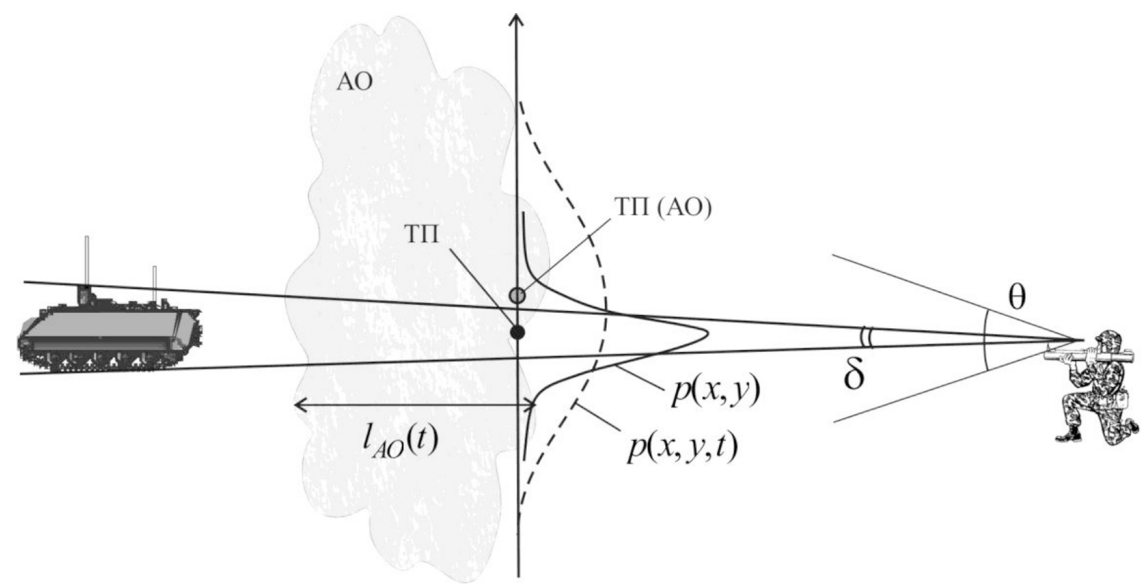

Рис. 1. К постановке задачи

Fig. 1. To the problem statement

по цели, полностью прикрытой аэрозольным образованием, пропускные свойства которого изменяются со временем - можно выделить этапы формирования, существования и рассеивания $\mathrm{AO}$ [3]. В качестве показателя эффективности ведения стрельбы примем вероятность попадания снаряда в цель $P_{n}$.

\section{Моделирование процесса стрельбы}

Динамику процесса стрельбы можно представить в виде ориентированного графа состояний, показанного на рис. 2 , где приняты следующие обозначения: $\mathrm{C}_{1}-$ исходное состояние; $\mathrm{C}_{2}$ - состояние, при котором в результате выстрела из гранатомета снаряд попал в цель; $\mathrm{C}_{3}-$ coстояние, при котором в результате выстрела из гранатомета снаряд не попал в цель. Принятие решения о попадании или непопадании снаряда в цель может осуществляться по дополнительно проявляющимся после выстрела признакам, например по звуку взрыва.

Каждый из переходов из состояния $i$ в состояние $j$ будет характеризоваться вероятностью и плотностью вероятности перехода $P_{i j}, \varphi_{i j}(t)$.

В соответствии с рекомендациями, изложенными в [7], представим динамику рассматриваемого процесса в форме передаточных функций (рис. 3). 


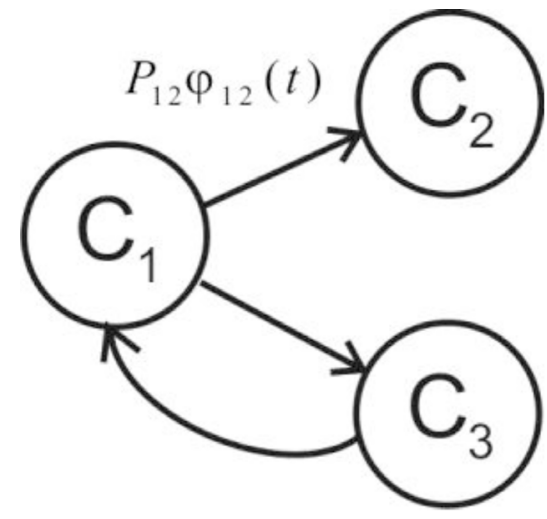

Рис. 2. Граф состояний

Fig. 2. State graph

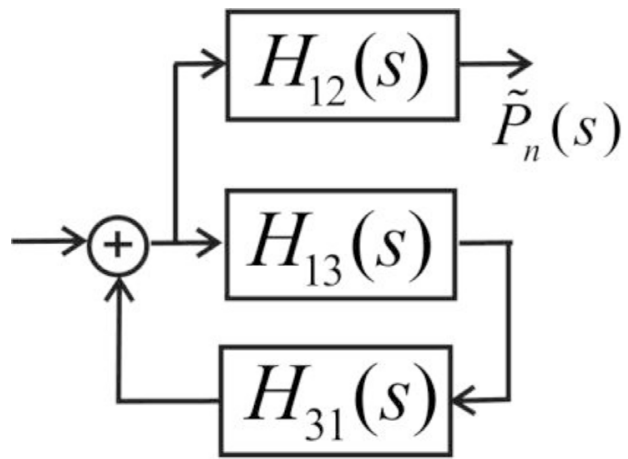

Рис. 3. Процесс стрельбы в форме передаточных функций

Fig. 3. Shooting process in the form of transfer functions

$H_{i j}(s)=\operatorname{Ls}\left[P_{i j} \varphi_{i j}(t)\right]$ - передаточная функция, представляющая собой преобразование Лапласа от произведения вероятности перехода на плотность вероятности времени перехода из состояния $i$ в состояние $j$.

Используя схему на рис. 3 , производя в соответствии с методами теории автоматических систем [8] несложные математические преобразования, можно записать следующее выражение для определения передаточной функции процесса стрельбы:

$$
H_{n}(s)=\frac{H_{12}(s)}{1-H_{13}(s) H_{31}(s)} .
$$

Введем в рассмотрение цикличность стрельбы, состоящую из $n$ итераций. Применение к (1) Z-преобразования дает [7]

$$
H_{n}(z, s)=\frac{H_{12}(s)}{1-z H_{13}(s) H_{31}(s)} .
$$

С учетом циклов по $n$ можно записать

$$
H_{n}(n, s)=H_{12}(s)\left[H_{13}(s) H_{31}(s)\right]^{n} .
$$

Тогда вероятность события, состоящего в том, что стрелком к некоторому моменту времени $t$ будет совершено ровно $n$ выстрелов и произойдет попадание в цель, можно записать следующим образом:

$$
P_{n}(n, t)=L_{s}^{-1}\left\{\frac{1}{s} H_{12}(s)\left[H_{13}(s) H_{31}(s)\right]^{n-1}\right\} .
$$

Вероятность попадания в цель в зависимости от числа прицельных выстрелов $n$ по ней одним стрелком однотипным оружием можно получить с использованием выражения

$$
P_{n}(N, t)=\sum_{n=1}^{N} P_{n}(n, t)
$$


Для случая, когда плотности вероятности распределения времени нахождения в одном состоянии при переходе в другое состояние определяются экспоненциальными распределениями, передаточные функции переходов можно записать следующим образом:

$$
H_{12}(s)=P_{12} \frac{\lambda_{12}}{s+\lambda_{12}} ; H_{13}(s)=\left(1-P_{12}\right) \frac{\lambda_{13}}{s+\lambda_{13}} ; H_{31}(s)=1,
$$

где $\lambda_{13}=\lambda_{12}-$ интенсивности стрельбы.

Интенсивность стрельбы связана со средним временем стрельбы обратной зависимостью $\lambda=1 / \bar{\tau}[9]$.

Для определения среднего времени стрельбы можно воспользоваться подходом, описанным в [9].

Среднее время стрельбы будет обусловлено в основном средним временем подготовки оружия к стрельбе, средним временем принятия решения о наличии цели и направлении стрельбы в сложных условиях обстановки, средним временем прицеливания и средним временем полета снаряда $\bar{\tau}_{12}=\bar{\tau}_{\text {поде }}+\bar{\tau}_{n p н}+\bar{\tau}_{n p}+\bar{\tau}_{n c}$.

При определении средних времен и переходных вероятностей $P_{12}$ и $P_{13}=1-P_{12}\left(P_{31}=1\right)$ необходимо учитывать следующее допущение двух ситуаций, когда стрелок потенциально может увидеть цель (этапы формирования или рассеивания АО) и когда цель визуально обнаружена быть не может (АО сформировано). Во втором случае определение направления стрельбы осуществляется на основе априорной информации о цели, полученной до постановки АО, ориентированием стрелка по шуму техники (на ее акустическое излучение), по формируемому в поле зрения изображению АО.

\section{Определение вероятности попадания в цель}

Вероятность попадания в цель можно определить как

$$
P_{12}=P_{n}(t \rightarrow \infty)=P_{\text {поде }}(t) P_{n p н \mid \text { поде }}(t) P_{n p \mid \text { прн }}(t),
$$

где $P_{\text {nодг }}(t)$ - вероятность подготовки оружия к стрельбе в зависимости от времени; $P_{n p н \mid \text { под }}(t)-$ условная вероятность принятия решения о наличии цели (обнаружение) или направлении стрельбы в условиях, когда цель не видна из-за АО, в зависимости от времени;

$$
\begin{aligned}
& P_{\text {прн/подг }}(t)=P_{\text {обн }}(t), \text { при } t \leq \bar{\tau}_{\text {необ; }} ; \\
& P_{\text {прн/иодг }}(t)=P_{\text {напр }}(t), \text { при } t>\bar{\tau}_{\text {необ }},
\end{aligned}
$$

где $P_{\text {обн }}(t)$ - вероятность обнаружения цели в зависимости от времени; $P_{\text {напр }}(t)-$ вероятность принятия решения о направлении стрельбы в условиях постановки АО в зависимости от времени; $\bar{\tau}_{\text {необ }}-$ среднее время принятия решения стрелком о необнаружении цели; $P_{n p \mid n p н}(t)-$ условная вероятность прицеливания и попадания в цель в зависимости от времени.

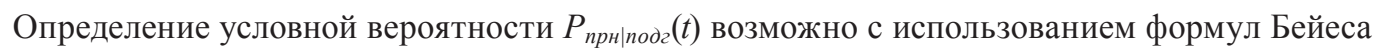
и полной вероятности [11].

Для случая формирования гипотез $H_{1}$ (стрелок определил направление стрельбы по цели в результате ее обнаружения) и $H_{2}$ (стрелок определил направление стрельбы по цели наугад (цель не обнаружена)), а также рассмотрения события $A$, заключающегося в том, что за время 
принятия решения о направлении стрельбы стрелок определит направление стрельбы по цели, можно записать следующее выражение:

$$
P_{\text {прн|поде }}(t)=P_{\text {оби }} P\left(H_{1} \mid A\right)+P_{\text {напр }} P\left(H_{2} \mid A\right),
$$

где $P\left(H_{1} \mid A\right)=\frac{P_{\text {обн }} P\left(H_{1}\right)}{P_{\text {обн }} P\left(H_{1}\right)+P_{\text {напр }} P\left(H_{2}\right)} ; P\left(H_{2} \mid A\right)=\frac{P_{\text {напр }} P\left(H_{2}\right)}{P_{\text {обн }} P\left(H_{1}\right)+P_{\text {напр }} P\left(H_{2}\right)}-$ апостериорные вероятности гипотез; $P\left(H_{1}\right)$ и $P\left(H_{2}\right)$ - вероятности гипотез.

Условную вероятность прицеливания и попадания в цель получим из следующих соображений.

Ошибки прицеливания, неоднообразные прикладка и удержание оружия, различные метеоусловия обуславливают ошибки выстрелов и вызывают рассеяние точек попадания [5]. Распределение точек попадания при ведении прицельной стрельбы по цели обычно характеризуют нормальным законом распределения [10]. При этом математические ожидания и средние квадратические отклонения (СКО) координат точек попадания ввиду вышеуказанных факторов будут нестационарными величинами и обуславливаться, например, сносом АО и изменением наблюдаемого стрелком фонового изображения ввиду флуктуаций $\mathrm{AO}$, опытностью и памятью стрелка.

Тогда двумерную плотность вероятности распределения координат точек попадания $x$ и $y$ как функцию времени можно записать как [11]

$$
p(x, y, t)=\frac{1}{2 \pi \sigma_{x}(t) \sigma_{y}(t)} \exp \left\{-\frac{\left[x-m_{x}(t)\right]^{2}}{2 \sigma_{x}^{2}(t)}-\frac{\left[y-m_{y}(t)\right]^{2}}{2 \sigma_{y}^{2}(t)}\right\}
$$

где $m_{x}(t), m_{y}(t)$ - математические ожидания координат точек попадания как функции времени; $\sigma_{x}(t), \sigma_{y}(t)$ - средние квадратические отклонения координат точек попадания в зависимости от времени; $\sigma_{x}(t)=\sqrt{\sigma_{n p}^{2}+\sigma_{n}^{2}+\sigma_{a}^{2}+\sigma_{m y}^{2}(t)}, \sigma_{n p}$ - ошибка прицеливания; $\sigma_{n}-$ ошибка, обусловленная опытностью и памятью стрелка; $\sigma_{a}$ - ошибка, обусловленная ориентированием стрелка по шуму техники (на ее акустическое излучение); $\sigma_{m y}(t)$ - ошибка, обусловленная метеоусловиями, флуктуационными свойствами $\mathrm{AO}$; для $\sigma_{y}(t)$ можно раскрыть аналогично.

С учетом «фигурности» реального объекта поражения (цели) условная вероятность прицеливания и попадания в цель в условиях ее прикрытия АО может быть записана следующим образом [11]:

$$
P_{n p \mid n p H}(t)=K_{\phi} \iint_{S_{o n}} p(x, y, t) d x d y,
$$

где $K_{\phi}=S_{u} / S_{\text {on }}$ - коэффициент фигурности [5]; $S_{u}$ - площадь цели; $S_{o n}=x_{u} y_{u}$ - площадь описанного вокруг цели прямоугольника; $x_{u}$ и $y_{u}$ - размеры цели по горизонтали и вертикали соответственно.

\section{Учет влияния нестационарного аэрозольного образования на процесс визуального обнаружения стрелком цели}

Предположим, что постановка АО осуществлена в момент подготовки стрелка к выстрелу.

Изменение пропускных для оптического излучения свойств среды принято характеризовать коэффициентом пропускания, определяемым показателем ослабления $k$ и оптической толщей $l_{A O}$, которая будет изменяться со временем (рис. 1) $[1,2,12] T(t)=\exp \left[-k \cdot l_{A O}(t)\right]$.

$$
-887-
$$


Как показано в [13], для случайного визуального просмотра заданного сектора поиска характерна экспоненциальная зависимость вероятности обнаружения от времени. При этом ввиду необходимости учета влияния нестационарных аэрозольных маскирующих помех можно воспользоваться результатами, полученными в $[2,3,9]$.

$$
P_{\text {обн }}(t, T)=1-\exp \left\{-\int_{T_{0}(0)}^{T(t)} \lambda[\varphi(x), x] \cdot \frac{d \varphi(x)}{d x} \cdot d x\right\},
$$

где $\lambda[\varphi(x), x]-$ интенсивность поиска, как функция времени и коэффициента пропускания; $\varphi(T)$ - функция, обратная $f(t)\left(t=\varphi(T), T_{t}^{\prime}=1 /[d \varphi(T) / d T]\right)$.

Выражение (12) представляет собой зависимость вероятности обнаружения цели от времени при случайном просмотре сектора поиска с учетом интенсивности поиска и задаваемого закона изменения коэффициента пропускания аэрозольного образования.

В процессе этапов формирования, существования и рассеивания АО, прикрывающего цель, стрелок перед каждым последующим выстрелом будет осуществлять процедуру поиска цели. Тогда с учетом параметров АО ( $\gamma_{1}$ и $\gamma_{3}-$ интенсивности формирования и рассеивания АО; $T_{0}$ и $T_{\min }$ - максимальное и минимальное значения коэффициента пропускания AO; $t_{1}$ и $t_{2}-$ моменты времени существования $\mathrm{AO}$ ) выражения для определения вероятностей обнаружения цели к моменту времени $t$ для случаев ведения поиска на этих этапах запишем следующим образом:

1) для этапа формирования $t<t_{1}$

$$
P_{\text {обн }}(t, T)=1-\exp \left\{-\int_{T_{0}}^{T(t)} \frac{\lambda(x)}{\gamma_{1} \cdot\left(T_{\min }-x\right)} d x\right\} ;
$$

2) для этапа существования $t_{1} \leq t<t_{2}$

$$
P_{\text {обн }}(t, T)=1-\exp \left\{-\int_{T\left(t_{1}\right)}^{T(t)} \lambda(x) \frac{d \varphi_{2}(x)}{d x} d x\right\} ;
$$

3) для этапа рассеивания $t \geq t_{2}$

$$
P_{\text {обн }}(t, T)=1-\exp \left\{-\int_{T\left(t_{2}\right)}^{T(t)} \frac{\lambda(x)}{\gamma_{3} \cdot\left(T_{0}-x\right)} d x\right\} .
$$

Выражение для определения интенсивности случайного визуального поиска получим, используя [13].

Предположим, что изначально в исходном состоянии стрелку уже известно местоположение цели и ему необходимо подготовиться, прицелиться и выстрелить. Учитывая возникшие в результате постановки АО сложные условия наблюдения, стрелок перед каждым выстрелом вынужден осуществлять поиск цели, находящейся в некоторой области неопределенности, обусловленной наиболее вероятным нахождением ее по представлению стрелка. Сектор поиска в этом случае значительно сужается. Поиск и прицеливание могут осуществляться с помощью прицельной планки (невооруженным глазом) или оптического прицела. На типовых дальностях применения гранатомета поле зрения оптического прицела соизмеримо с полем поиска, поэтому процедура поиска может осуществляться без сканирования прицелом (поиск будет 
осуществляться в поле зрения оптического прицела глазом стрелка или невооруженным глазом в равнозначном поле поиска через прицельную планку).

Интенсивность случайного поиска невооруженным глазом протяженного одиночного неподвижного объекта можно определить следующим образом [13]:

$$
\lambda(x=T(t))=\frac{C[K T(t)]^{2} \delta^{3}\left[L_{\phi} T(t)\right]^{0,3}}{\theta^{2}},
$$

где $C$ - коэффициент, характеризующий индивидуальные способности наблюдателя (для монокулярного зрения $-C_{M}=12$ град ${ }^{2}$ угл. мин $\left.{ }^{-3}\left(\kappa д / \mathrm{M}^{2}\right)^{-0,3} \mathrm{c}^{-1}\right) ; K=\left(L_{\phi}-L_{o}\right) / L_{\phi}-$ яркостный контраст объекта; $L_{\phi}$ и $L_{o}-$ яркости фона и объекта [кд/м²]; $\delta$ - угловой размер объекта [угл. мин]; $\theta$ - угловой размер поля поиска [град].

При поиске в оптический прицел яркость фона, угловой размер объекта и контраст рассчитывают по формулам [13]: $L_{\phi}^{\prime}=\tau_{o} L_{\phi} ; \delta^{\prime}=\Gamma \delta ; K^{\prime}=K /(1+q)$, где $\tau_{o}-$ коэффициент светопропускания; $\Gamma$ - увеличение оптического прицела; $q$ - коэффициент светорассеяния.

Среднее время принятия решения о наличии цели и направлении стрельбы в условиях нестационарной аэрозольной маскирующей помехи с вероятностью $P_{\text {обн }}\left(\bar{\tau}_{\text {необ }}\right)$ может быть определено следующим образом:

$$
\begin{aligned}
& \bar{\tau}_{\text {прн }}=\bar{\tau}_{\text {обн }}, \text { при } t \leq \bar{\tau}_{\text {необ}} ; \\
& \bar{\tau}_{\text {прн }}=\bar{\tau}_{\text {пор }}+\bar{\tau}_{\text {напр }}, \text { при } t>\bar{\tau}_{\text {необ }},
\end{aligned}
$$

где $\bar{\tau}_{\text {обн }}=\int_{0}^{\infty} t \cdot \frac{\frac{d}{d t} P_{\text {обн }}(t, T)}{P_{\text {обн }}(t \rightarrow \infty)} d t-$ среднее время принятия решения об обнаружении цели с вероятностью $P_{\text {обн }}\left(\bar{\tau}_{\text {необ }}\right)$.

\section{Результаты апробации методики}

$\mathrm{C}$ целью апробации предлагаемой методики были произведены расчеты для следующих исходных данных: начало системы координат совпадает с центром цели; размеры цели $x_{u}=3 \mathrm{M}$; $y_{u}=2 \mathrm{м} ; K_{\phi}=0,83 ; \sigma_{x}=\sigma_{y}=0,5$ м; на дальности порядка 200 м $\delta=42$ угл. мин.; $L_{\phi}=30$ кд/м²; $K=0,17$; поле зрения оптического прицела $\theta=13$ град; $\Gamma=2,7 ; q=0,05 ; \tau_{o}=0,6 ; \bar{\tau}_{12}=12$ с (средняя скорострельность РПГ-7 составляет 5 выстрелов/мин.); $P_{\text {поде }}=0,98 ; P_{n p \mid n p н}=0,79 ; P_{\text {напр }}=0,14$; $\bar{\tau}_{\text {необ }}=4 \mathrm{c} ; \gamma_{1}=2,8 \mathrm{c}^{-1} ; \gamma_{3}=0,1 \mathrm{c}^{-1} ; T_{0}=0,95 ; T_{\min }=0,004 ; t_{1}=3 \mathrm{c} ; t_{2}=32 \mathrm{c} ;$ время постановки АО $2,8 \mathrm{c}$.

На рис. 4 представлены графики зависимости условной вероятности прицеливания и попадания боеприпаса в цель в некоторый момент времени $t_{0} P_{n p \mid n p t}\left(t_{0}, \sigma_{x}\right)$ (в соответствии с выражением (10)) от ошибок прицеливания по оси $x$ для различных значений нестационарных $m_{x}(t), m_{y}(t)$

На рис. 5 представлены результаты расчетов зависимости вероятности обнаружения цели невооруженным глазом и через оптический прицел от времени для трех этапов существования АО. Анализ полученных результатов показывает, что на этапе формирования АО при некоторых исходных данных может возникать ситуация, когда обнаружение цели стрелком осуществляется практически мгновенно с вероятностью, близкой единице. Это объясняется

$$
-889-
$$




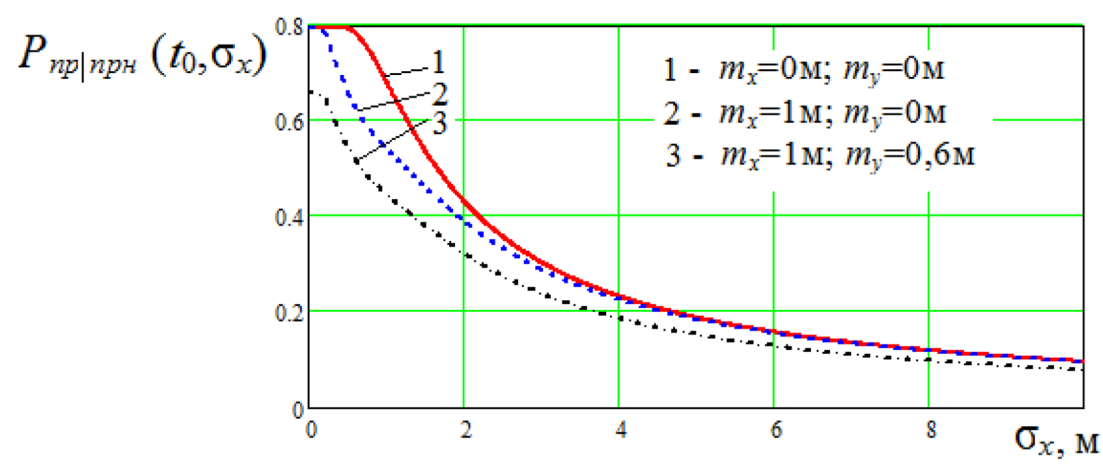

Рис. 4. Графики зависимости условной вероятности прицеливания и попадания боеприпаса в цель от ошибок прицеливания по оси $x$ в момент времени $t_{0}$

Fig. 4. Dependence plots of conditional aiming probability and ammunition hitting the target from aiming errors along the $\mathrm{x}$-axis at the time $t_{0}$

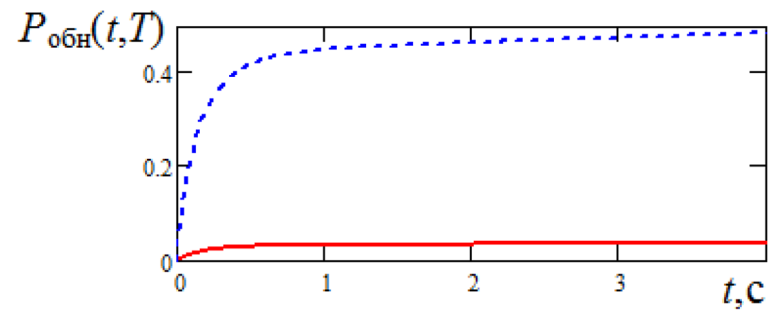

a) на этапе формирования $\mathrm{AO}$

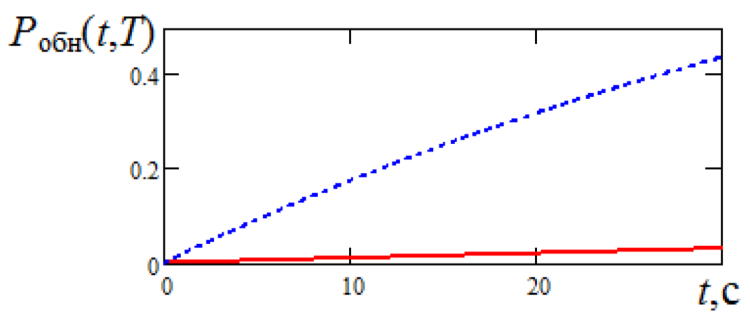

б) на этапе существования АO

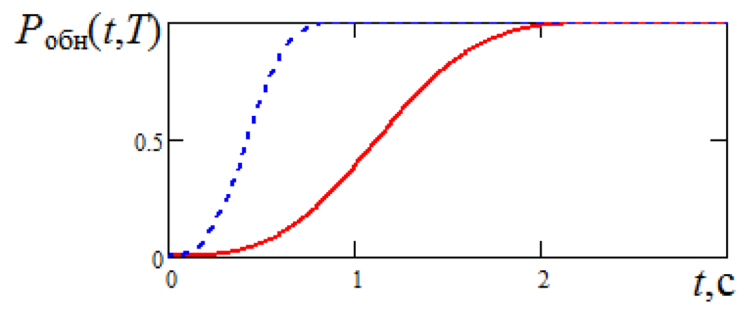

в) на этапе рассеивания $\mathrm{AO}$

Рис. 5. Графики зависимости вероятности обнаружения цели невооруженным глазом (-) и через оптический прицел (--) от времени для трех этапов существования АО

Fig. 5. Target detection probability graphs with the naked eye (-) and through the telescopic sight (--) from time to time for three stages of existence AF 
тем, что поле поиска практически совпадает с полем зрения прицела, а в случае с оптическим прицелом - еще и с увеличением видимых размеров объекта. Поэтому время обнаружения в этом случае может ограничиваться временем реакции глаза человека (порядка 0,1-1 с [13]). В представленном случае вероятность обнаружения к моменту времени $\bar{\tau}_{\text {необ }}=4$ с не превышает 0,035 и 0,485 для поиска невооруженным глазом и через оптический прицел соответственно.

На втором этапе существования АО $\left(t_{1} \leq t<t_{2}\right)$ обнаружение цели как невооруженным глазом, так и через оптический прицел возможно с малой вероятностью. На третьем этапе среднее время обнаружения при указанных исходных данных составило 1,27 и 0,49 с невооруженным глазом и через оптический прицел соответственно.

В соответствии с выбранным показателем и полученным для его определения выражением (4) построены графики зависимости вероятности попадания $n$-м выстрелом в цель от времени (рис. 6) и график распределения финальной вероятности того, что при совершении ровно $n$ выстрелов произойдет попадание в цель (рис. 7). Анализ результатов показывает, что в интервалы времени, когда АО еще не сформировано или рассеивается, вероятность попадания существенно выше.

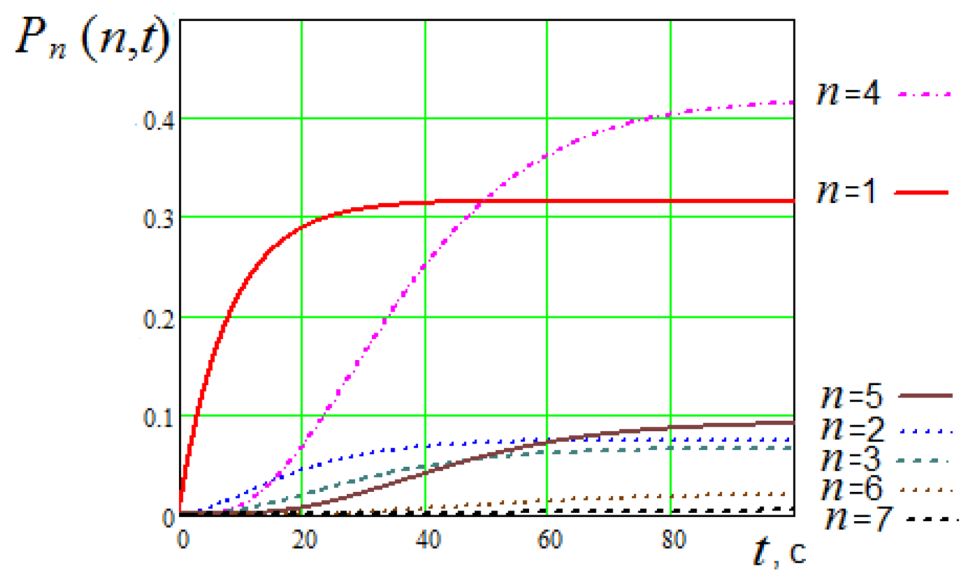

Рис. 6. Графики зависимости вероятности попадания $n$-м выстрелом в цель от времени

Fig. 6. Graphs of the dependence of the probability of hitting the target with the $n$-th shot on time

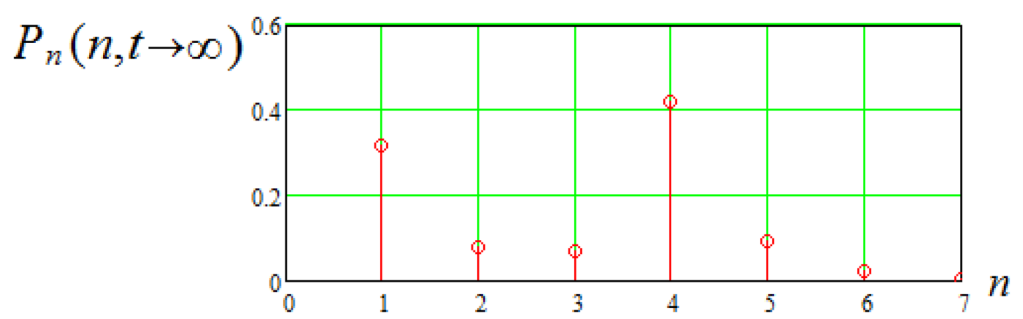

Рис. 7. График распределения финальной вероятности того, что при совершении ровно $n$ выстрелов произойдет попадание в цель

Fig. 7. The graph of the distribution of the final probability of that when exactly $n$ shots are fired, the target will be hit 


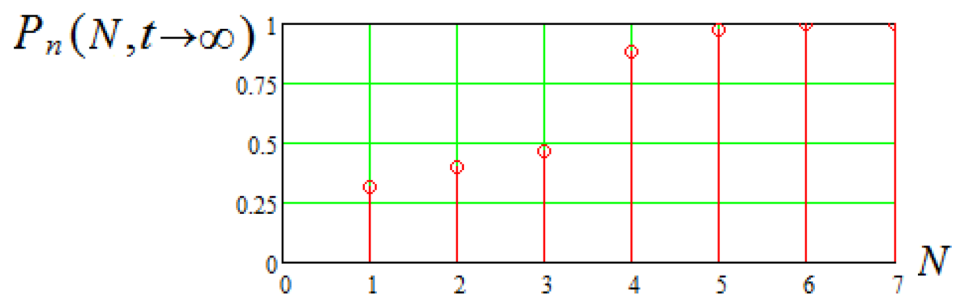

Рис. 8. График распределения финальной вероятности попадания в цель от числа выстрелов

Fig. 8. The graph of the distribution of the final probability of hitting the target on the number of shots

На рис. 8 представлена зависимость финальной вероятности попадания боеприпаса в цель от числа прицельных выстрелов по ней одним стрелком, однотипным оружием в условиях прикрытия цели нестационарным аэрозольным образованием.

Анализ полученных результатов показывает адекватность разработанной методики изменяемым исходным данным (в том числе нестационарным числовым характеристикам координат точек попадания боеприпаса, обусловленным совокупностью вышеописанных факторов).

\section{Заключение}

Таким образом, с использованием теории графов, теории вероятностей, методов преобразования Лапласа разработана методика оценки эффективности применения аэрозольных образований для защиты объектов от прицельной стрельбы из гранатомета. Методика позволяет получать вероятностные показатели попадания снарядов в цель при стрельбе из гранатомета в сложных условиях обстановки с учетом временных характеристик процесса стрельбы и совокупности ошибок прицеливания, обусловленных рядом факторов, в том числе применением аэрозольных завес. Использование математического аппарата производящих функций позволило определить вероятностные показатели процесса стрельбы с учетом цикличности, обусловленной количеством выстрелов по цели.

\section{Список литературы / References}

[1] Нестеровский И.П., Тосенко В.М. Экспериментальные исследования флуктуации оптической толщины искусственных аэрозольных образований, Радиотехника, 1997, 6, 106-108 [Nesterovsky I.P., Tosenko V.M. Experimental studies of fluctuations in the optical thickness of artificial aerosol formations, Radiotechics, 1997, 6, 106-108 (in Russian)].

[2] Козирацкий Ю.Л. Поиск цели оптико-электронными системами в условиях нестационарных атмосферных аэрозольных помех, Радиотехника, Информационный конфликт в спектре электромагнитных волн, спеи. выпуск, 1995, 88-89 [Koziratsky Yu.L. Search for a target by optoelectronic systems in conditions of non-stationary atmospheric aerosol interference, Radiotechics, Information conflict in the spectrum of electromagnetic waves, special. issue, 1995, 6, 88-89 (in Russian)].

[3] Козирацкий А.Ю., Федукович З.Б., Прохоров Д.В., Бурзак И.В. Поиск цели оптико-электронными средствами в условиях нестационарных маскирующих помех, Радиотехника, 2005, 7, 63-65 [Koziratsky Yu.L., Fedukovich Z.B., Prokhorov D.V., Burzak I.V. Target search by optical- 
electronic means in conditions of non-stationary masking interference, Radiotechics, 2005, 7, 63-65 (in Russian)].

[4] Козирацкий А.Ю., Прохоров Д.В., Кулешов П.Е., Федукович З.Б. Учет стохастичности характера формирования структуры аэрозольного образования в моделях поиска цели оптико-электронными средствами, Радиотехника, 2006, 9, 78-80 [Koziratsky A.Yu., Prokhorov D.V., Kuleshov P.E., Fedukovich Z.B., Taking into account the stochasticity of the nature of the formation of the structure of aerosol formation in models of target search by optoelectronic means, Radiotechics, 2006, 9, 78-80 (in Russian)].

[5] Наставление по стрелковому делу. Основы стрельбы из стрелкового оружия, М.: Военное издательство МО РФ, 1970. 176 с. [Manual on shooting/ Fundamentals of shooting from small arms, M.: Military publishing house of the Ministry of Defense of the Russian Federation, 1970. 170 p. (in Russian)].

[6] Наставление по стрелковому делу. Ручной противотанковый гранатомет (РПГ-7 и РПГ-7Д), М.: Военное издательство МО РФ, 1983. 152 с. [Manual on shooting. Hand-held anti-tank grenade launcher (RPG-7 and RPG-7D), M.: Military publishing house of the Ministry of Defense of the Russian Federation, 1983. 152 p. (in Russian)].

[7] Козирацкий Ю.Л., Козирацкий А.Ю., Прохоров Д.В. и др. Модели информационного конфликта средств поиска и обнаружения. Монография, М.: Радиотехника, 2013. 232 с. [Kоzіratsky Yu.L., Koziratsky A.Yu., Prokhorov D.V. and others. Models of information conflict of search and detection tools. Monograph, M.: Radiotechics, 2013. 232 p. (in Russian)].

[8] Зайцев Г.Ф. Теория автоматического управления и регулирования, Киев: Высш. шк. Головное изд-во, 1989. 431 с. [Zaitsev G.F. Theory of automatic control and regulation, Kiev: Higher shk. Head publishing house, 1989. 431 p. (in Russian)].

[9] Козирацкий Ю.Л., Козирацкий А.Ю., Прохоров Д.В. и др. Модели пространственного и частотного поиска. Монография, М.: Радиотехника, 2013. 344 с. [Koziratsky Yu.L., Koziratsky A.Yu., Prokhorov D.V. and others. Spatial and frequency search models. Monograph, M.: Radiotechics, 2013. 344 p. (in Russian)].

[10] Бобриков А.А., Авотынь Б.А., Анисимов Е.Г. и др. Оченка эффективности огневого поражения ударами ракет и огнем артиллерии: военно-теоретический труд, СПб.: Галея Принт, 2006. 421 c. [Bobrikov A.A., Avotyn B.A., Anisimov E.G. and others. Evaluation of the effectiveness of fire damage by missile strikes and artillery fire: military theoretical work, St. Petersburg: Galeya Print, 2006. 421 p. (in Russian)].

[11] Шторм Р. Теория вероятностей. Математическая статистика. Статистический контроль качества, М.: Мир, 1970. 368 с. [Storm R. Probability theory. Mathematical statistics. Statistical quality control, M.: Mir, 1970. 368 p. (in Russian)].

[12] Зуев В.Е. Лазер-метеоролог, Л.: Гидрометеоиздат, 1974. 180 c. [Zuev V.E. Laser meteorologist, L.: Gidrometeoizdat, 1974. 180 p. (in Russian)].

[13] Травникова Н.П. Эффективность визуального поиска, М.: Машиностроение, 1985. 128 c. [Travnikova N.P. The effectiveness of visual search, M.: Mechanical engineering, 1985. 128 p. (in Russian)]. 